\title{
'|||||||||||||||||||||||||||||||||||||||||||||||||||||||||||||||||||.
}

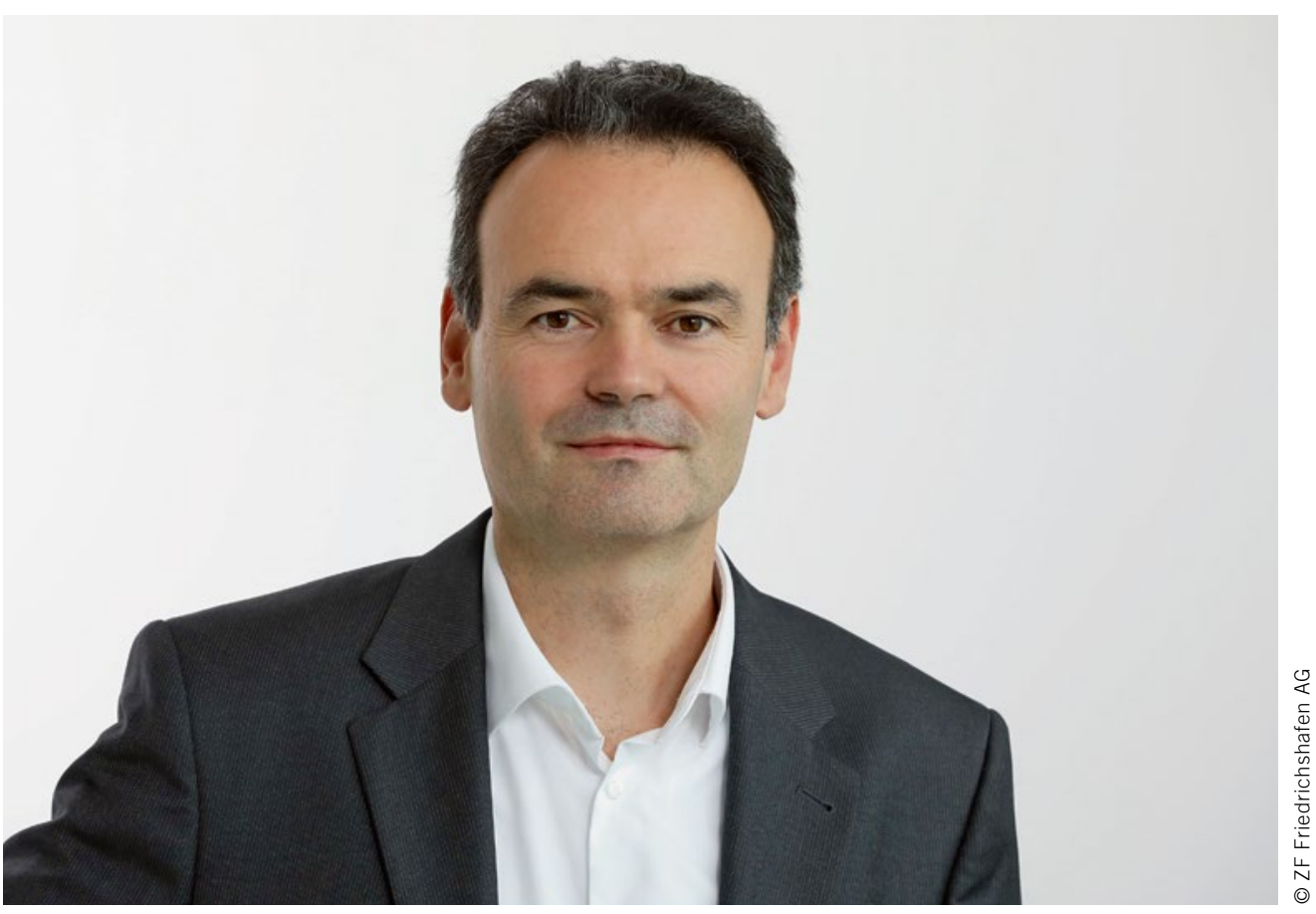

Dr. Otmar Scharrer

Senior Vice President R\&D

ZF Friedrichshafen AG

\section{Electromobility Enters the Decisive Phase}

Electromobility today appears in various forms. The PHEV concept is of great importance for the near future. Only this type of driveline concept is currently capable of ideally combining customer requirements in a single car: electric and thus locally emission-free driving in the city with sufficient range on long-distance journeys. A prerequisite is a sufficiently large battery storage system, which will be available in the vast majority of next generation hybrid vehicles. ZF presented this concept under the name EVplus with a guaranteed electric range of $80 \mathrm{~km}$ as a fully functional test vehicle some years ago.

When it comes to BEV, a number of variants are in the field and many more are currently under development. The currently available technical diversity is striking. This is already true for the electric motor, where the entire range of possibilities is determined by various motor designs and winding types of the electrical machine. But it also applies to power electronics, where numerous different power modules are now installed at different voltage levels. The number of variants is likely to increase even further by the application of silicon carbide technology. Finally, there are coaxial as well as parallel axis systems in a wide variety of installations as single or multiple units. For manufacturers as well as suppliers, the question therefore arises as to whether standardization makes sense.
The OEMs will certainly conduct a make-or-buy analysis in individual cases and thus determine their technological footprint. For the suppliers, on the other hand, it is very important to make a technological leap at the right time, in which as many existing innovations as possible are embedded in a new, modular concept in which individual features can also be realized for specific customer requirements.

In most parts of the world, the automotive industry is currently developing the last generation of drives with an internal combustion engine. This will give a strong impetus to electromobility. The Covid-19 pandemic was one of the main reasons for the further acceleration of these activities. Electromobility is therefore entering the decisive phase in which it will replace combustion engine mobility. The key to success will be not only the costs but also the attainable range for a given battery size. In addition to the hardware solutions, next-generation software components that are critical to success will also come onto the market, which will make a major contribution to efficiency through predictive estimation of traffic situations. Additional functions for the customer, such as vehicle-to-grid, round off the range of this next BEV generation. In this phase, the system concept will become more important than ever, in order to be able to adapt to changing boundary conditions at the required speed. 